\title{
Emotion recognition and cognitive empathy deficits in adolescent offenders revealed by context-sensitive tasks
}

\section{Maria Luz Gonzalez-Gadea ${ }^{1,2,3+}$, Eduar Herrera' ${ }^{1,2,4}{ }^{+}$, Mario Parra $3,5,6,7$, Pedro Gomez Mendez ${ }^{8}$, Sandra Baez ${ }^{1,2,3}$, Facundo Manes ${ }^{1,2,3,9}$ and Agustin Ibanez ${ }^{1,2,3,4,9}$ *}

1 Laboratory of Experimental Psychology and Neuroscience, Institute of Cognitive Neurology, Buenos Aires, Argentina

${ }^{2}$ National Scientific and Technical Research Council, Buenos Aires, Argentina

${ }^{3}$ UDP-INECO Foundation Core on Neuroscience, Diego Portales University, Santiago, Chile

${ }^{4}$ Universidad Autonoma del Caribe, Barranquilla, Colombia

${ }^{5}$ Human Cognitive Neuroscience, Psychology Department, University of Edinburgh, Edinburgh, UK

${ }^{6}$ Scottish Dementia Clinical Research Network, Perth, UK

${ }^{7}$ Neuropsy and Biomedical Unit, Health School, University Surcolombiana, Neiva, Colombia

${ }^{8}$ Universidad del Norte, Barranquilla, Colombia

${ }^{9}$ Centre of Excellence in Cognition and its Disorders, Australian Research Council, Sydney, NSW, Australia

\section{Edited by:}

John J. Foxe, Albert Einstein College of Medicine, USA

\section{Reviewed by:}

Inti Brazil, Donders Institute for Brain, Cognition and Behaviour, Netherlands Patricia Lockwood, University College London, UK

Peter Vermeulen, Autisme Centraal, Belgium

\section{${ }^{*}$ Correspondence:}

Agustin Ibanez, Laboratory of Experimental Psychology and Neuroscience, Institute of Cognitive Neurology, Pacheco de Melo 1854/60, C1126AAB Buenos Aires, Argentina e-mail: aibanez@ineco.org.ar

${ }^{\dagger}$ Maria Luz Gonzalez-Gadea and Eduar Herrera are first authors.
Emotion recognition and empathy abilities require the integration of contextual information in real-life scenarios. Previous reports have explored these domains in adolescent offenders (AOs) but have not used tasks that replicate everyday situations. In this study we included ecological measures with different levels of contextual dependence to evaluate emotion recognition and empathy in AOs relative to non-offenders, controlling for the effect of demographic variables. We also explored the influence of fluid intelligence (FI) and executive functions (EFs) in the prediction of relevant deficits in these domains. Our results showed that AOs exhibit deficits in context-sensitive measures of emotion recognition and cognitive empathy. Difficulties in these tasks were neither explained by demographic variables nor predicted by FI or EFs. However, performance on measures that included simpler stimuli or could be solved by explicit knowledge was either only partially affected by demographic variables or preserved in AOs. These findings indicate that AOs show contextual social-cognition impairments which are relatively independent of basic cognitive functioning and demographic variables.

\section{Keywords: offenders, adolescence, delinquency, social cognition, contextual processing, ecological tasks}

\section{INTRODUCTION}

Adolescent offenders (AOs) are known to present with difficulties in emotion recognition and empathy. However, the nature of such affective impairments is still a matter of debate. While both emotion recognition and empathy require implicit integration of contextual social cues in complex environments, most tasks used to assess these domains in AOs can be solved through the application of abstract rules and explicit knowledge of social norms. In addition, performance on these tasks is thought to partially reflect the influence of basic cognitive skills, such as intellectual ability or executive functions (EFs). We propose that a more realistic approach to explore these difficulties may be afforded by context-sensitive and ecologically valid measures. In the present study, we investigated emotion recognition and empathy in AOs and non-offenders using tasks with different levels of contextual dependence and involvement of real-life scenarios. We also explored the impact of fluid intelligence (FI) and EFs on emotion recognition and empathy difficulties.

Facial emotion recognition is a context-sensitive process influenced by visual information, voices, bodies, and even words (Barrett etal., 2011; Ibanez etal., 2014b). Such a process is impaired in AOs (McCown et al., 1986; Jones et al., 2007; Marsh and Blair, 2008; Fairchild et al., 2009; Sato et al., 2009), as shown through tasks using static facial stimuli with a limited range of emotional expressions. Evidence from adult offenders suggests that difficulties in these tasks may result from confounding factors, such as low education or poor verbal IQ (Glass and Newman, 2006; Pham and Philippot, 2010; Domes et al., 2013).

AOs also exhibit impairments of empathy (Ellis, 1982; Burke, 2001; Lindsey et al., 2001; Jolliffe and Farrington, 2004; Decety et al., 2009; de Wied et al., 2012; Domes et al., 2013). This complex, context-sensitive domain (Melloni et al., 2014) refers to the capacity to share and understand the subjective experience of others in reference to oneself (Decety et al., 2012b). It involves both affective (sharing and responding to the emotional experience of others) and cognitive (understanding the intentions and perspectives of others) components. Some studies with both young and adult offenders have reported greater difficulties in cognitive than in affective empathy (Jolliffe and Farrington, 2004). However, more recent reports of adolescents and adults with marked psychopathic/antisocial traits (Jones et al., 2010; Schwenck et al., 2012; Lockwood et al., 2013a; Pasalich et al., 2014) revealed the opposite profile (i.e., more deficits in affective than cognitive components of empathy). Despite the complexity of empathy deficits in AOs, traditional studies have examined the issue using only selfreport questionnaires, yielding mixed results. While some studies 
found significant differences between AOs and non-offenders (Ellis, 1982; Burke, 2001; Lindsey et al., 2001), others reported similar results for both groups (Kaplan and Arbuthnot, 1985; Lee and Prentice, 1988; Bush etal., 2000; Domes et al., 2013). Finally, studies in adult offenders found that the relationship between low empathy and offending behavior seems to be affected by IQ (Jolliffe and Farrington, 2004) and education (Domes et al., 2013). Empathy failures among adults and young offenders may also reflect executive dysfunction (Jolliffe and Farrington, 2004), although this proposal has not been tested heretofore.

Taken together, these studies suggest that factors such as IQ, education, and underlying cognitive functions may account both for deficits in emotion recognition and empathy in AOs and for the inconsistencies found in the literature. In several reports, AOs' low verbal IQ was shown to tamper their general ability to solve cognitive tasks (Isen, 2010; Frisell et al., 2012) and was associated with low education level (Isen, 2010; Mottus et al., 2012). Likewise, there is evidence for the role of low IQ in EF deficits (Jolliffe and Farrington, 2004; Parra et al., 2005; Koolhof et al., 2007; Kennedy et al., 2011; Frisell et al., 2012).

Whereas verbal IQ depends on previous knowledge, FI reflects an individual's capacity for abstract thought and reasoning (Catell, 1971). FI modulates affective and social cognition (Ibanez et al., 2013, 2014b). Although no previous study has assessed FI abilities in AOs, a recent report (Huepe et al., 2011) has found an association between low FI and poor psychosocial adaptation in adolescents under vulnerability conditions. However, to our knowledge, no previous study has investigated the differential contribution of FI and EFs to emotion recognition and empathy in AOs.

An important step to properly explore such issues is to acknowledge that both emotion recognition and empathy abilities require the integration of contextual cues in real-life scenarios (Ibáñez and Manes, 2012; Kennedy and Adolphs, 2012; Melloni et al., 2014). Nevertheless, most tasks used to assess these domains in AOs can be solved with explicit knowledge and fail to emulate the emotions and behaviors presented in real-life social interactions. As an alternative, we propose that difficulties in emotion recognition and empathy may be better understood through the use of ecologically valid, context-sensitive tasks requiring implicit inference of contextual cues (Baez et al., 2012, 2013, 2014c; Baez and Ibanez, 2014). Indeed, these tasks have proven sensitive to impairments in everyday activities in psychiatric populations (Burgess et al., 1998; Torralva et al., 2009a; Ibáñez and Manes, 2012; Melloni et al., 2014). Given that AOs manifest disruptive behavior and severe difficulties in daily social interactions (Carswell et al., 2004; Jolliffe and Farrington, 2004; Pardini and Fite, 2010), we suggest that context-sensitive measures may provide a more realistic approach to identify emotion recognition and empathy profiles in AOs.

Furthermore, recent evidence (Glass and Newman, 2009; Baskin-Sommers et al., 2011, 2012) suggests that emotional processing deficits in psychopathic individuals are explained by difficulties in processing contextual information. Particularly, a recent study on this population (Brazil et al., 2013) reported difficulties in the processing of contextual cues when this had to be done in an effortful way, with intact automatic use of cue-related information. We propose that AOs may exhibit similar difficulties, leading to failures in context-sensitive tasks.

In the present study we explore the performance of AOs in emotion recognition and empathy tasks involving real-life scenarios and two levels of contextual dependence. To this aim, we included: (i) emotion recognition tasks with low Emotional Morphing Task (EMT) and high The Awareness of Social Inference Test (TASIT) and Dual Valence Association Task (DVAT) context processing requirements, and (ii) two measures of empathy, namely, a self-report questionnaire Interpersonal Reactivity Index (IRI) with low contextual dependence and an ecologically valid task with high contextual integration demands Empathy for Pain Task (EPT). We have previously shown the high and low contextual dependence and involvement of real-life scenarios of these paradigms (Baez et al., 2012, 2013, 2014c; Baez and Ibanez, 2014). Here, we compared performance on these tasks between AOs and non-offenders while controlling for the effect of demographic variables (education and age). We also explored whether FI and EFs could partially predict the deficits of AOs in these domains. We hypothesize that AOs will exhibit primary deficits in emotion recognition and empathy tasks with high context-processing requirements. Conversely, we predict that their performance in tasks with low context-sensitivity will be preserved or explained by demographic or cognitive variables.

\section{MATERIALS AND METHODS PARTICIPANTS}

Forty-six male participants (30 AOs and 16 non-offenders) were enrolled in the present study. The AOs were recruited from a reform school of young male offenders in Barranquilla, Colombia. According to file records, most of the AOs were recidivist (74\%) and had been incarcerated between 4 and 48 months following murder $(35 \%)$ or robbery $(65 \%)$ sentences. The AOs completed a structured admission interview, aimed to confirm that they did not fulfill criteria for a life-time diagnosis of psychiatric disorders and were not under pharmacological treatment during the assessment. Although most AOs had a history of drug and/or alcohol use, none was diagnosed with addiction or was treated for this reason.

Non-offenders were recruited from higher schools located in the same district of residence of AOs. Recruitment was authorized and assisted by the schools' principals and teachers. Inclusion criteria for control participants were: (a) gender (male), (b) age (between 15 and 18 years old), (c) education level (less than 12 years of education), and (d) absence of history of psychiatric or neurological disorders. All the adolescents provided informed assent and a parent or guardian provided informed consent in agreement with the Helsinki declaration. The study was approved by the ethics committee of CARI Mental Hospital of Barranquilla, Colombia.

\section{INSTRUMENTS}

\section{Emotion recognition assessment}

Low context-sensitive measure: facial emotion recognition. We assessed facial emotion recognition using the EMT, which consists of photos of facial expressions featuring six basic emotions 
(happiness, surprise, sadness, fear, anger, and disgust). These images, taken from the Pictures of Affect Series (Ekman and Friesen, 1976), were morphed for each prototype emotion and for a neutral state (Young et al., 1997). The shape and texture differences between a neutral image $(0 \%)$ and a full emotion face $(100 \%)$ were manipulated in increments of 5\% (500 ms for each image). The 48 morphed facial stimuli were randomly presented on a computer screen. Participants were asked to press a button as soon as they recognized the facial expression and then to identify it from a forced-choice list of six options. The images remained visible until the participant responded. We measured the mean accuracy of overall emotion recognition (maximum one point) and the accuracy for each emotion category. This task has been previously validated (Young et al., 1997) and used with other neuropsychiatric populations (Baez et al., 2012, 2013, 2014c; Couto et al., 2013; Cardona et al., 2014).

High context-sensitive measure: contextual inference of emotional states. We assessed contextual inference of emotional states through The Awareness of Social Inference Test (TASIT). The TASIT is a validated clinical test of social perception (McDonald etal., 2006) and has been extensively used to evaluate contextual inference of emotions in different neuropsychiatric disorders (Rankin et al., 2009; Baez et al., 2012, 2013, 2014c; Couto et al., 2013). This task requires time-efficient processing of contextual cues (e.g., prosody, facial movement, and gestures) taxing selective attention and social reasoning. Such demands are absent in tasks involving static stimuli. Specifically, we used part 1 of the TASIT - the emotion evaluation test (EET) -, which comprises 20 short (15-60 s-long) videotaped vignettes of trained professional actors interacting in everyday situations. After viewing each scene, participants must choose the emotion expressed by the focused actor (fear, surprise, sadness, anger, disgust) from a forced-choice list. A global score was calculated from the sum of accurately recognized trials (maximum 20 points) and for each emotional state (maximum four points for each one).

High context-sensitive measure: emotional interference. We also included a Dual Valence Association Task (DVAT) to measure emotion recognition under interference effects produced by a double categorization of valences and stimuli. This is a validated task (Ibanez et al., 2011) based on implicit association principles (Greenwald et al., 1998; Ibáñez, 2010). Participants must classify faces and words as either positive or negative by pressing a left or right key, respectively. The stimulus set includes pictures of happy and angry faces, and pleasant and unpleasant words. There are 10 stimuli per category. The task is organized in two blocks of 35 randomized trials in which words and faces are alternately presented for 300 and $100 \mathrm{~ms}$, respectively. In congruent trials, stimuli must be categorized as angry-unpleasant (left) or happy-pleasant (right). In incongruent trials, the words must be categorized in the same manner while face categories appear on the opposite side of the screen in angry-pleasant (left) or happy-unpleasant (right) configurations. Thus, the latter trials require participants to inhibit the implicit contextual association of both emotional valence categories (e.g., a happy face with a pleasant word). A DVAT score was calculated for each subject based by subtracting mean reaction times of congruent blocks from those of incongruent blocks. In addition, we calculated the mean accuracy of both congruent and incongruent blocks (maximum 35 points).

\section{Empathy assessment}

Low context-sensitive instrument: self-report questionnaire. We assessed empathy through the IRI, a validated self-report questionnaire (Davis, 1983) extensively used for research on AOs (Jolliffe and Farrington, 2004; Lovett and Sheffield, 2007). The IRI includes 28 items that separately measure the cognitive and affective components of empathy. The instrument contains four scales: perspective taking (PT), empathic Concern (EC), Fantasy $(\mathrm{F})$, and personal distress $(\mathrm{PD})$.

High context-sensitive measure: ecological/behavioral task. We also administered an adaptation of an EPT previously validated with behavioral measures, eye-tracking, and fMRI (Decety et al., 2012a). It has been used to evaluate empathy deficits in psychopathic offenders (Decety et al., 2013), adolescents with psychopathic traits (Marsh et al., 2013), adolescents with antecedents of social deprivation (Escobar et al., 2014), and children with conduct disorders (Lockwood et al., 2013b). Our adapted version has been used in the assessment of other neuropsychiatric populations (Baez et al., 2012, 2013, 2014a,c; Couto et al., 2013; Baez and Ibanez, 2014). This task assesses empathy in the context of intentional and accidental harm. The task consists of 25 animated scenarios (11 intentional, 11 accidental, 3 neutral) involving two individuals. Each scenario consists of three digital color pictures presented in succession to imply motion. Three types of situations are depicted: (i) intentional harm, in which one person is in a painful situation intentionally caused by another (e.g., purposely stepping on someone's toe); (ii) accidental harm, where one person is in a painful situation accidentally caused by another; and (iii) control or neutral situations (e.g., one person receiving a flower from another).

Since the protagonists' faces were not visible, participants could not rely on them to guess emotional reactions. However, body expressions and postures provided sufficient information about the emotional reaction of the victim and the intention of the agent. Participants were asked to respond to three pair's different questions. The first pair addressed cognitive aspects of empathy, namely (a) intentionality (Was the action done on purpose?) and (b) intention of the perpetrator to hurt the victim (How bad was the purpose?). The second pair tapped affective aspects, namely, (c) emphatic concern (How sad do you feel for the victim?), and (d) degree of discomfort (How upset do you feel for what happened in the situation?). The third pair assessed moral evaluation, namely (e) correctness of the action (How inappropriate was the action?), and (f) punishment (How much penalty does this action deserve?). The question about the intentionality of the action was answered by selecting Yes/No. The other questions were answered using a computer-based visual analog scale (ranging from -9 to 9 ), whose numbers were not visible to participants. The meaning of the scale's extreme values depends on the question. For example, for the question "How sad do you feel for the hurt person?," one extreme of the bar reads "I feel very sad" and the other one reads "I do not feel sad at all." Performance was assessed considering 
the percentage of accuracy for the intentionality question and the ratings for the other questions.

\section{Fluid intelligence}

The Raven's Standard Progressive Matrices (RSPM; Raven et al., 2008) was used as a measure of FI. Participants completed a series of drawings by considering the spatial organization of an array of objects, identifying relevant features, and choosing one object that matched one or more of the identified features.

\section{Executive functions}

We used the Institute of Cognitive Neurology (INECO) Frontal Screening test (IFS; Torralva et al., 2009b), which assesses frontal lobe function as indexed by the following subtasks: motor programming, conflicting instructions, Verbal Inhibitory Control, Abstraction Ability (proverbs interpretation), Backward Digit Span, Spatial Working Memory, and Go/NoGo. This task has been used with different neuropsychiatric populations (Torralva et al., 2009b, 2012; Fiorentino et al., 2013; Baez et al., 2014b) A mean total score is calculated from the sum of the subtask scores $(30$ points). A 25-point cutoff score has shown a sensitivity of $96.2 \%$ and a specificity of $91.5 \%$ in detecting patients with dysexecutive syndrome (Torralva et al., 2009b).

\section{DATA ANALYSIS}

One-way repeated measures analysis of variance (ANOVA) and Tukey's HSD post hoc tests were used (when appropriate) to analyze differences between groups in emotion recognition and empathy tasks. Demographic, FI, and EF data were compared between groups using student's $t$-tests. To control for the effect of demographic and cognitive variables on our experiments, we first matched the groups in term of education and FI. Second, we used age and years of education as covariate variables in an ANCOVA. We reported the effects both before and after covariation. Finally, we conducted multiple regression analyses to explore whether emotion recognition and empathy deficits were partially explained by FI and EFs. The emotion recognition and empathy measures that were significantly different between groups after covariance analyses were separately considered as dependent variables. Group, RSPM score, and total IFS score were included as predictors. The $\alpha$ value for all statistical tests was set at 0.05 . Eta squared $\left(n^{2}\right)$ was used as a measure of effect size for significant effects.

\section{RESULTS}

\section{DEMOGRAPHIC DATA}

Table 1 shows no significant group differences in education $\left[t_{(44)}=0.821, p=0.416\right]$. Although both groups had very similar age, significant differences were found $\left[t_{(44)}=-3.73, p=0.001\right]$ and further ANCOVA was performed to control this variable.

\section{GROUP DIFFERENCES IN EMOTION RECOGNITION AND EMPATHY Emotion recognition}

Low context-sensitive measure: facial emotion recognition. Relative to non-offenders, AOs had a significantly poorer performance on the $\operatorname{EMT}\left[F_{(1,44)}=9.61, p=0.003, n^{2}=0.17\right.$; see Figure 1A $]$. This effect persisted after co-varying for education $\left[F_{(1,43)}=8.99\right.$, $p=0.004, n^{2}=0.17$ ] but became marginally significant after
Table 1 | Demographic, neuropsychological and behavioral data.

\begin{tabular}{llll}
\hline & $\begin{array}{l}\text { Adolescent } \\
\text { offenders } \\
(\boldsymbol{N}=\mathbf{3 0})\end{array}$ & $\begin{array}{l}\text { Non-offenders } \\
(\boldsymbol{N}=\mathbf{1 6})\end{array}$ & $\boldsymbol{p}^{*}$ \\
\hline Age & $16.67(0.54)$ & $16(0.63)$ & 0.001 \\
Education (years) & $6.50(1.77)$ & $7.25(1.88)$ & 0.416 \\
Fluid intelligence (RSPM) & $48.53(4.51)$ & $49.44(4.38)$ & 0.517 \\
Executive functions (EFS) & & & \\
Motor programming & $2.70(0.46)$ & $3(0.00)$ & 0.014 \\
Conflicting instructions & $2.96(0.18)$ & $2.75(0.44)$ & 0.024 \\
Verbal inhibitory control & $3.86(1.79)$ & $5.50(0.73)$ & 0.001 \\
Abstraction (proverbs) & $1.40(0.89)$ & $2.81(0.40)$ & 0.000 \\
Backward digit span & $2.83(1.05)$ & $2.25(1.18)$ & 0.094 \\
Spatial working memory & $3.06(1.22)$ & $3.93(0.25)$ & 0.008 \\
Go/NoGo & $2.83(0.46)$ & $2.93(0.25)$ & 0.407 \\
IFS global score & $20.86(3.54)$ & $24.93(1.65)$ & 0.000 \\
\hline
\end{tabular}

* Student t-test. RSPM, Raven's Standard Progressive Matrices; IFS, INECO Frontal Screening.
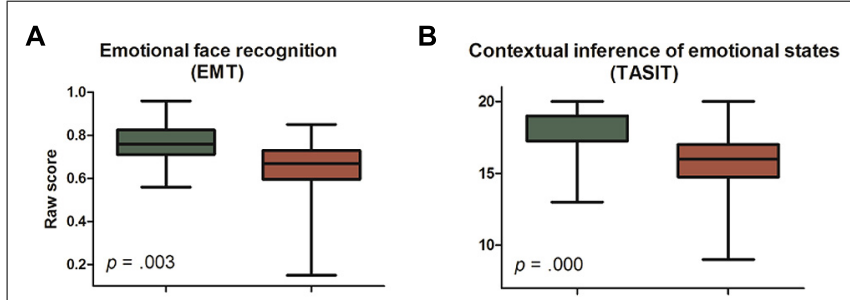

C
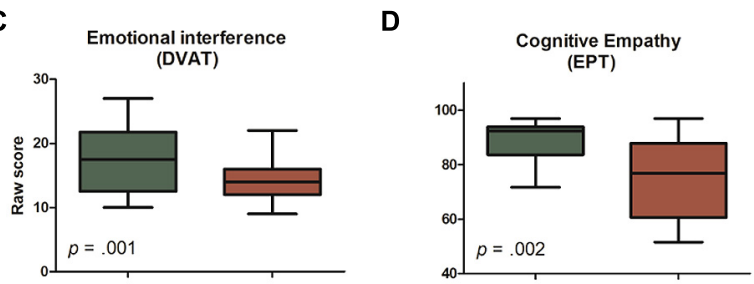

Controls

Offenders

FIGURE 1 | Significant group differences in emotion recognition and empathy tasks. (A) Emotional Morphing Task (EMT): mean accuracy and $\mathrm{SD}$ of emotion recognition. Note that group differences disappeared after adjusting for age (see Low Context-Sensitive Measure: Facial Emotion Recognition). (B) Awareness of Social Inference Test (TASIT): mean accuracy and SD for global score. (C) Dual Valence Association Task (DVAT): mean accuracy and SD for incongruent blocks. (D) Empathy for Pain Task (EPT): cognitive components (percentage and SD for intentionality question).

adjusting by age $\left[F_{(1,43)}=3.85, p=0.056, n^{2}=0.08\right]$. A repeated-measures analysis including type of emotions revealed no interaction between emotions and groups $\left[F_{(5,220)}=1.00\right.$, $p=0.417$; see details in Table 2].

High context-sensitive measure: contextual inference of emotional states. Significant group differences were found in the 
Table 2 | Means (SD) and group comparisons in the emotion recongnition and empathy task with low and high level of contextual dependence.

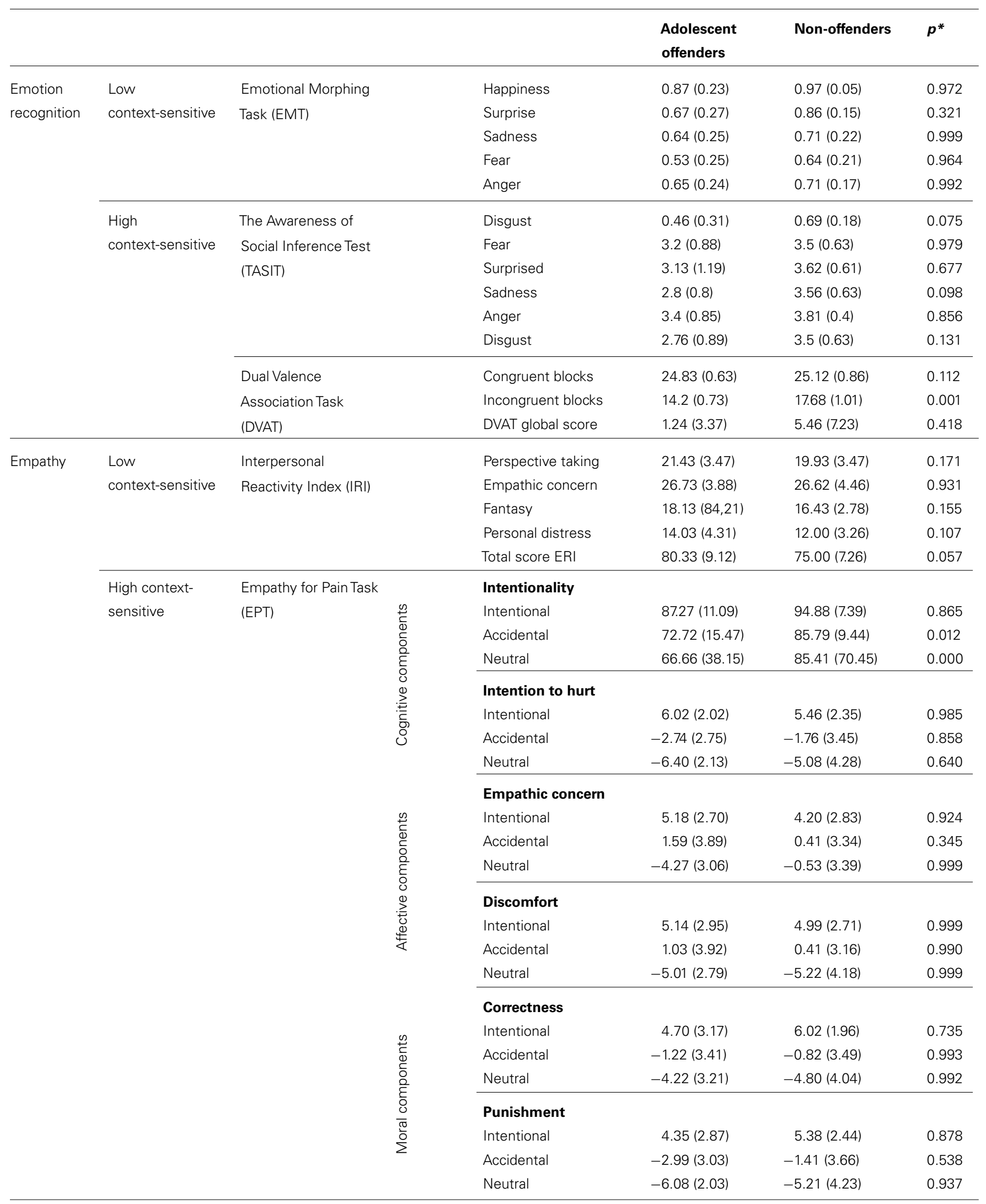

* Tukey post-hoc analyses between groups for each category. 
TASIT global score $\left[F_{(1,44)}=14.92, p=0.000, n^{2}=0.25\right]$, AOs identifying fewer emotions than non-offenders (see Figure 1B). This effect persisted after controlling for co-variables [education: $F_{(1,43)}=18.98, p=0.000, n^{2}=0.30$; and age: $F_{(1,43)}=10.35$, $\left.p=0.002, n^{2}=0.19\right]$. No interaction between groups and emotion categories were observed $\left[F_{(4,176)}=0.67, p=0.610\right.$; see details in Table 2].

High context-sensitive measure: emotional interference. A repeated-measures analysis with accuracy scores from the DVAT in congruent and incongruent blocks revealed a significant interaction between Group and Block $\left[F_{(1,44)}=4.62, p=0.036\right.$, $\left.n^{2}=0.09\right]$. A post hoc analysis (Tukey's HSD, $M S=14.13$, $d f=85.06)$ showed that AOs made more errors in incongruent blocks than non-offenders $(p<0.001$; see Figure 1C). These effects persisted after co-varying for education $\left[F_{(1,43)}=5.04\right.$, $\left.p=0.030, n^{2}=0.10\right]$ and age $\left[F_{(1,43)}=3.90, p=0.034\right.$, $\left.n^{2}=0.08\right]$. No significant differences between groups were found for the DVAT main score $\left[F_{(1,44)}=0.66, p=0.418\right.$; see details in Table 2].

In summary, AOs exhibited deficits in emotion recognition tasks. While difficulties in isolated emotion face recognition were partially mediated by age, deficits in contextual emotional inference as well as in emotional interference (both task requiring contextual integration of emotional information) were independent from co-variables.

\section{Empathy}

Low context-sensitive measure: self-report questionnaire. No significant differences between groups were found for IRI total score $\left[F_{(1,44)}=4.07, p=0.059\right]$ or any of its four subscales separately (see Table 2).

High context-sensitive measure: ecological/behavioral task. Regarding the cognitive components of empathy, AOs presented significantly poorer comprehension of the intentionality of pain situations than non-offenders $\left[F_{(1,44)}=10.97, p=0.002\right.$, $n^{2}=0.20$; see Figure 1D]. This effect was maintained after adjusting for co-variables [education: $F_{(1,43)}=10.09, p=0.003$, $n^{2}=0.19$; and age: $\left.F_{(1,43)}=5.39, p=0.020, n^{2}=0.11\right]$. However, no significant differences were observed in ratings of intention to hurt $\left[F_{(1,44)}=0.93, p=0.338\right]$. Regarding the affective components, no group differences were found in empathic concern $\left[F_{(1,44)}=1.45, p=0.234\right]$, or in the degree of discomfort $\left[F_{(1,44)}=0.21, p=0.649\right]$. No significant differences were observed in terms of moral aspects of empathy, either in the correctness-of-action $\left[F_{(1,44)}=0.38\right.$, $p=0.554]$ or the punishment $\left[F_{(1,44)}=3.01, p=0.089\right]$ ratings. Finally, no significant interactions were found between Group and Situation (intentional, accidental, and neutral) across the different components of empathy (see details in Table 2).

In sum, AOs showed impairments in the cognitive components of empathy, as assessed by the context-sensitive task. Specifically, they had difficulties to identify intentionality in situations in which someone suffers harm (EPT). No significant differences were observed in the self-report measure of empathy (IRI).

\section{INDIVIDUAL DIFFERENCES IN FI AND EFs}

No significant group differences were found in FI $\left[t_{(44)}=0.653\right.$, $p=0.517$; see Table 1]. However, relative to non-offenders, AOs showed significantly poorer performance on EFs [IFS global score: $t_{(44)}=4.33, p=0.0001$ ] and on most of the IFS sub-scales [motor programming: $t_{(44)}=2.56, p=0.014$; conflicting instructions: $t_{(44)}=-2.33, p=0.024$; verbal inhibitory control: $t_{(44)}=3.47$, $p=0.001$; abstraction: $t_{(44)}=5.97, p=0.0001$; and spatial working memory $\left.t_{(44)}=2.78, p=0.008\right]$. No significant differences were observed in the backward digit span $\left[t_{(44)}=-1.71\right.$, $p=0.094]$ and Go/NoGo $\left[t_{(44)}=0.83, p=0.407\right]$ subtasks.

\section{ARE EMOTION RECOGNITION AND EMPATHY DEFICITS PARTIALLY EXPLAINED BY FI OR EFs?}

Multiple regression analyses were performed to evaluate the influence of FI and EFs on the emotion recognition and empathy impairments observed in Group Differences in Emotion Recognition and Empathy section. Figure 2 shows that none of the dependent variables were predicted by FI or EFs (see more results in Table 3).

\section{DISCUSSION}

In this study we employed a range of context-sensitive ecological measures to examine the emotion recognition and empathy

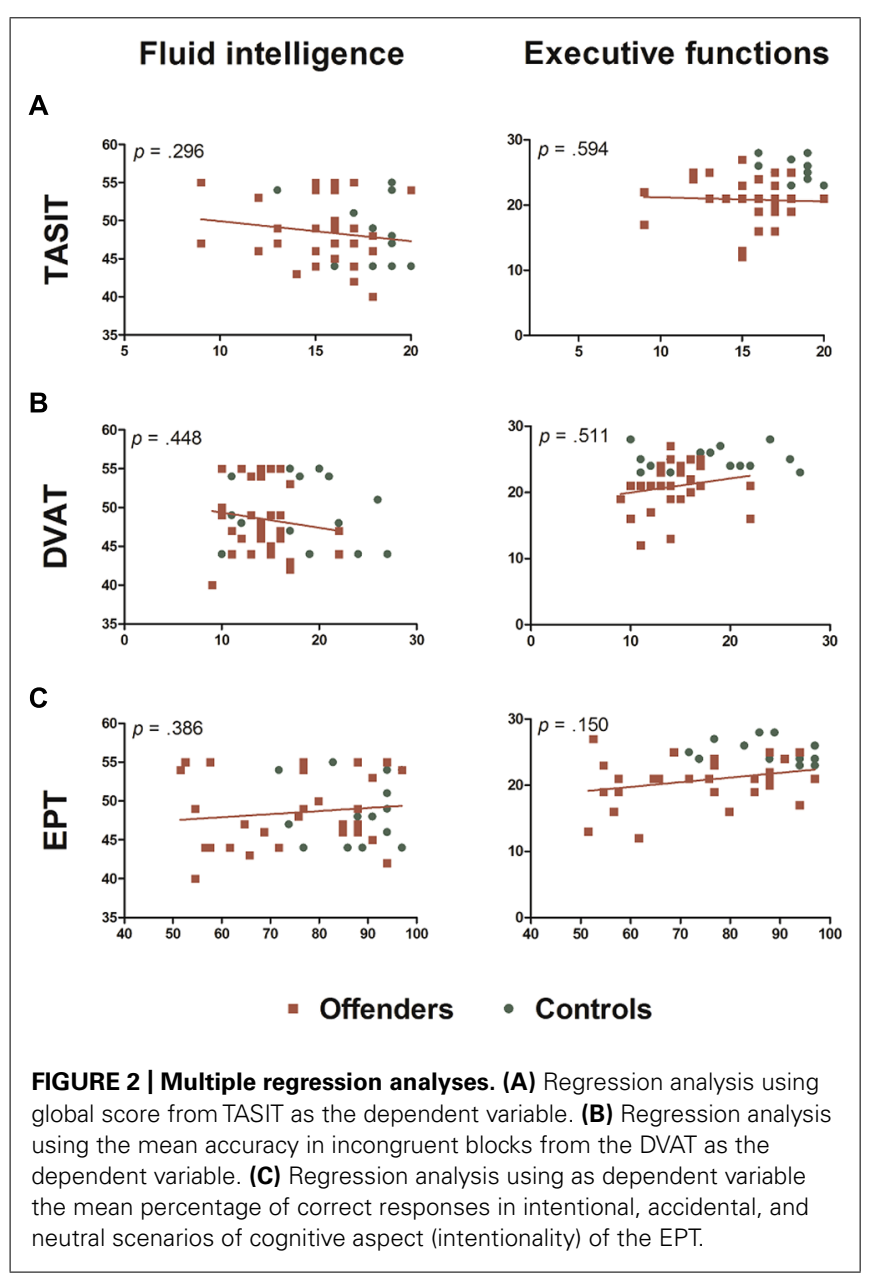


Table 3 | Coefficients of the multiple regression models of results reported in "Are Emotion Recognition and Empathy Deficits Partially Explained by FI or EFs?" section.

\begin{tabular}{|c|c|c|c|c|c|c|}
\hline & \multicolumn{2}{|c|}{$\begin{array}{l}\text { Contextual inference of } \\
\text { emotional states (TASIT) }\end{array}$} & \multicolumn{2}{|c|}{$\begin{array}{l}\text { Emotional Interference } \\
\text { (DVAT) }\end{array}$} & \multicolumn{2}{|c|}{ Cognitive empathy (EPT) } \\
\hline & $\beta$ & $p$ & $\beta$ & $p$ & $\beta$ & $p$ \\
\hline Fluid intelligence (RSPM) & -0.14 & 0.296 & -0.11 & 0.448 & -0.12 & 0.386 \\
\hline Executive Functions (IFS) & -0.08 & 0.524 & -0.11 & 0.511 & -0.23 & 0.150 \\
\hline
\end{tabular}

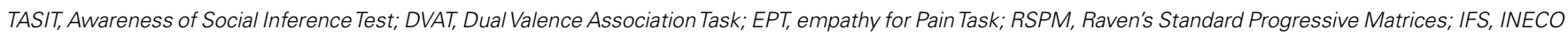
Frontal Screening.

profiles of AOs. Crucially, we controlled for the influence of demographic variables, such as age and education, and investigated the influence of FI and EFs on task performance. Our results showed that difficulties in tasks requiring contextual appraisal (TASIT, DVAT, and EPT) were not explained by covariates. However, performance on measures that included more simple stimuli or could be solved by explicit knowledge was either partially affected by demographic variables (EMT) or preserved in AOs (IRI). This study provides preliminary evidence that AOs exhibit social contextual processing impairments which are relatively independent from education, FI, or EFs.

\section{EMOTION RECOGNITION AND EMPATHY DEFICITS IN AOs}

Previous studies have shown impairments in affective processing in AOs, specifically in the recognition of negative emotions (such as anger, disgust, fear, or sadness) during facial recognition tasks (McCown et al., 1986; Jones et al., 2007; Sato et al., 2009). In our study, we explored this domain through the EMT which includes a dynamic method for the presentation of facial expressions. The results thus obtained showed that AOs have a general difficulty in emotion recognition in the EMT, regardless of emotion type. However, these differences become marginally significant after adjusting for age. A previous study (Pham and Philippot, 2010) using a similar EMT with adult offenders found that deficits in decoding emotional facial expression were accounted for by education. In our study, AOs and non-offenders were matched by education, but group differences were found in age. Several studies (Thomas etal., 2007; Mancini etal., 2013) have reported that face emotion recognition abilities develop with age, with adults displaying more sensitivity to subtle changes in emotional expression than adolescents (Thomas et al., 2007). Therefore, differences in age affected performance on the EMT, possibly due to general effects of neurocognitive development. Taken together, previous and present results suggest that demographic variables, such as education and age, should be controlled for in order to unveil difficulties in basic emotion processing in AOs.

Moreover, emotion recognition difficulties in AOs were revealed by the TASIT, which requires the integration of cues from face, prosody, gesture, and social context to identify emotions (Baez et al., 2012, 2013; Baez and Ibanez, 2014). The age variable partially affected facial emotion recognition deficits, but it had no effect on the difficulties to infer more complex affective states. A previous study (Kipps et al., 2009) has demonstrated that contextual cues in the TASIT normally lead to more accurate emotion identification in healthy individuals. We suggest that performance on the TASIT may depend more on contextual integration skills than in basic facial emotion recognition abilities, which are associated with developmental trajectories (Thomas et al., 2007; Mancini et al., 2013).

We also found emotional interference difficulties in AOs assessed by the DVAT. Based on implicit association principles (Greenwald etal., 1998), this task assesses the interference effect produced by the implicit contextual association of categories with incongruent emotional valence (e.g., an angry face with a pleasant word). The performance on the DVAT requires the integration of emotional valence from facial expressions and semantic information in a highly associative context (Ibanez et al., 2011, 2014a). Our results showed that AOs were impaired at automatically discriminating two contextual opposed valences. This could be triggered by basic impairments of emotional binding processes, inhibitory control to deal with the interference effects in incongruent blocks, or a combination of both factors (Ibanez et al., 2011). We support the view that the AOs' difficulties revealed by the DVAT and TASIT could be explained by a general impairment in integration of emotion and context.

Finally, to evaluate empathy we included both an ecological behavioral measure of empathy (the EPT) and a self-report questionnaire (the IRI). AOs showed deficits in the cognitive components of the EPT, but demonstrated no difficulties in the IRI. These behavioral results suggest affective-processing difficulties in AOs which are not revealed by self-report questionnaires. The IRI considers empathy as a trait and fails to fully represent empathic abilities because of its limited ecological validity (Ickes, 2009). Furthermore, AOs showed deficits in the cognitive components of empathy assessed by the EPT - specifically, in the comprehension of deliberately harmful actions. These deficits remained after covarying for demographic variables and were not predicted by FI or EFs.

It is important to note that the attribution of the action's intentionality in the EPT is the main goal of the task and it is crucial to respond correctly to the affective and moral aspects associated with the actions observed in the task (Baez et al., 2013, 2014b). No difficulties were observed in the affective aspects of empathy. 
This result is consistent with previous findings suggesting that offending is more strongly associated with low cognitive empathy than low affective empathy (Jolliffe and Farrington, 2004). In addition, a recent study (Pasalich et al., 2014) found that callousunemotional traits in adolescents with conduct problems were uniquely associated with deficits in cognitive empathy. Note, however, that most studies on adolescents with psychopathy or conduct disorders have reported greater deficits in the affective than in the cognitive aspects of empathy (Jones et al., 2010; Schwenck et al., 2012; Lockwood et al., 2013a). We suggest that both empathic processes can be difficult to separate, since the understanding of a cognitive aspect usually implies affective processing, and vice versa. This overlap between cognitive and affective components may partially explain the inconsistencies found in the literature and should be addressed by future studies.

In addition, the EPT results revealed that AOs had no difficulties to judge the correctness of actions performed by the perpetrator or the punishment deserved. This finding is consistent with previous evidence for adequate moral judgment in offenders (Cima et al., 2010; Schmoll, 2012; Radke et al., 2013).

In summary, we confirmed our prediction that emotion recognition and empathy deficits in AOs were described by tasks involving real-life scenarios and/or implicit contextual information (TASIT, DVAT, and EPT). These deficits were neither explained by demographic variables nor predicted by cognitive functioning. However, performance on the facial emotion recognition task (EMT), which has lower context-processing demands, was affected by age. In the same vein, AOs gave no signs of empathy deficits in the IRI questionnaire, probably due to the involvement of more explicit knowledge of social norms which would be preserved in these adolescents (Cima et al., 2010; Radke et al., 2013).

\section{DEFICITS IN PROCESSING CONTEXTUAL INFORMATION IN AOS}

The present results suggest that AOs have difficulties in integrating affective processes with contextual information in ecological tasks. Although to our knowledge no previous study has addressed offender's deficits to process contextual information, recent studies in psychopaths have begun to explore these difficulties within this population (Newman et al., 2010; Baskin-Sommers et al., 2013; Sadeh etal., 2013). These studies found that emotional deficits in psychopaths are moderated by difficulties to focus attention in complex scenarios. Thus, these authors proposed that information processing deficits in psychopathy could be explained by the interplay between attentional and emotional systems.

In the present study we found EFs impairments in AOs. The task used to assess such functions is known to tax top-down attentional function integrated in the frontal lobe. Although we failed to find associations between such tasks and measures of contextual processing in the assessed sample, such an association may still exist. For example, the ecological nature of the tasks used to assess contextual-dependent emotional and empathy processing may pose different demands on attentional functions that might denote other cognitive processes in these individuals. Future studies in AOs should explore whether deficits in ecological tasks that require contextual integration could be explained by attentional deficits which are also measured with more ecologically valid procedures. For example, studies with psychopathic individuals should consider exploring difficulties in contextual information processing in social cognition by using similar context-sensitive measures.

\section{LIMITATIONS AND FUTURE DIRECTIONS}

Some important limitations of this study should be acknowledged. First, we did not include a measure of verbal IQ to control group differences between AOs and non-offenders. Low verbal IQ has been proposed as a confounding variable that may explain deficits in emotion recognition and empathy in adult offenders (Jolliffe and Farrington, 2004; Domes et al., 2013). However, we controlled the influence of education by selecting AOs and nonoffenders with similar education level and including this variable as a covariate. Recent reviews suggest that low verbal IQ may be a consequence of adolescents' truancy or verbal-educational deficits accumulated throughout childhood (Isen, 2010; Mottus et al., 2012). In other words, controlling education levels may indirectly control the effect of verbal IQ. Nevertheless, futures studies should consider including verbal IQ as a control measure of offenders' cognitive task performance.

Second, we used the IFS as a screening measure of EFs. This test has been employed in several neuropsychiatric populations (Torralva et al., 2009b; Gleichgerrcht et al., 2011; Fiorentino et al., 2013; Baez et al., 2014b). However, AOs are known to exhibit deficits in inhibitory control and working memory (Bergvall et al., 2001; Koolhof et al., 2007; Verona et al., 2012). It would be desirable for future examinations to clarify the influence of these domains on emotion recognition and empathy tasks.

Third, since psychopathic/callous-unemotional traits seem to capture meaningful heterogeneity in $\mathrm{AOs}$ at the behavioral and neural levels (Sebastian et al., 2012; Viding et al., 2012; Lockwood et al., 2013b), they may be related to the observed emotion recognition and empathy deficits. Future studies should assess and control for the impact of these traits on emotional and empathy deficits in AOs.

Fourth, some methodological issues must be contemplated in future studies. We used several statistical tests to compare groups in tasks with different levels of contextual dependence. More sophisticated methods should be used in future research, including a parametric classification of low and high context-sensitive measures into a single general linear model.

Finally, our sample size was relatively small and only included male AOs. However, our sample was arguably large enough for the type of analyses performed (Hair et al., 2010), and it was not smaller than those in previous studies with offenders (Sato et al., 2009; Domes et al., 2013). Further research should assess the effect of context processing in emotion recognition and empathy domains in larger samples of AOs and extend these results in female population.

\section{CONCLUSION}

Our study documents emotion recognition and empathy impairments in AOs. These are reflected by difficulties to integrate affective process (emotion and empathy) with contextual information in tasks that incorporate real-life scenarios. The results 
showed that AOs exhibit deficits in ecological, context-sensitive measures of emotion recognition and empathy (TASIT, DVAT, and EPT). Difficulties in these tasks were neither explained by demographic variables nor predicted by FI nor EFs. However, deficits in more basic emotion recognition tasks (EPT) were accounted for by age, while no difficulties were observed in measures that can be solved through explicit knowledge (IRI).

These results suggest that ecological measures are sensitive tools that should be applied in the assessment of AOs. Although implementation would be challenging, rehabilitation programs could aid AOs in the use of implicit rules to interpret contextual cues in real-life social environments.

\section{ACKNOWLEDGMENTS}

This research was partially supported by grants from CONICET, CONICYT/FONDECYT Regular (1130920 and 1140114), FONCyT-PICT 2012-0412 and 2012-1309, and INECO Foundation. The authors thank the Colombian Family Welfare Institute (ICBF) for helping with the patient recruitment process.

\section{REFERENCES}

Baez, S., Herrera, E., Villarin, L., Theil, D., Gonzalez-Gadea, M. L., Gomez, P., etal. (2013). Contextual social cognition impairments in schizophrenia and bipolar disorder. PLOS ONE 8:e57664. doi: 10.1371/journal.pone. 0057664

Baez, S., and Ibanez, A. (2014). The effects of context processing on social cognition impairments in adults with Asperger's syndrome. Front. Neurosci. 8:270. doi: 10.3389/fnins.2014.00270

Baez, S., Ibanez, A., Gleichgerrcht, E., Perez, A., Roca, M., Manes, F., et al. (2014a). The utility of the IFS (INECO Frontal Screening) for the detection of executive dysfunction in adults with bipolar disorder and ADHD. Psychiatry Res. 15, 269 276. doi: 10.1016/j.psychres.2014.01.020

Baez, S., Manes, F., Huepe, D., Torralva, T., Fiorentino, N., Richter, F., et al. (2014b). Primary empathy deficits in frontotemporal dementia. Front. Aging Neurosci. 6:262. doi: 10.3389/fnagi.2014.00262

Baez, S., Marengo, J., Perez, A., Huepe, D., Giralt-Font, F., Rial, V., et al. (2014c). Theory of mind and its relationship to executive functions and emotion recognition in borderline personality disorder. J. Neuropsychol. doi: 10.1111/jnp.12046 [Epub ahead of print].

Baez, S., Rattazzi, A., Gonzalez-Gadea, M., Torralva, T., Vigliecca, N., Decety, J., et al. (2012). Integrating intention and context: assessing social cognition in adults with Asperger syndrome. Front. Hum. Neurosci. 6:302. doi: 10.3389/fnhum.2012.00302

Barrett, L. F., Mesquita, B., and Gendron, M. (2011). Context in emotion perception. Curr. Dir. Psychol. Sci. 20, 286-290. doi: 10.1177/0963721411422522

Baskin-Sommers, A., Curtin, J. J., Li, W., and Newman, J. P. (2012). Psychopathyrelated differences in selective attention are captured by an early event-related potential. Personal Disord. 3, 370-378. doi: 10.1037/a0025593

Baskin-Sommers, A. R., Curtin, J. J., and Newman, J. P. (2011). Specifying the attentional selection that moderates the fearlessness of psychopathic offenders. Psychol. Sci. 22, 226-234. doi: 10.1177/0956797610396227

Baskin-Sommers, A. R., Curtin, J. J., and Newman, J. P. (2013). Emotion-modulated startle in psychopathy: clarifying familiar effects. J. Abnorm. Psychol. 122, 458468. doi: 10.1037/a0030958

Bergvall, A. H., Wessely, H., Forsman, A., and Hansen, S. (2001). A deficit in attentional set-shifting of violent offenders. Psychol. Med. 31, 1095-1105. doi: 10.1017/S0033291701004317

Brazil, I. A., Maes, J. H., Scheper, I., Bulten, B. H., Kessels, R. P., Verkes, R. J., et al. (2013). Reversal deficits in individuals with psychopathy in explicit but not implicit learning conditions. J. Psychiatry Neurosci. 38, E13-E20. doi: 10.1503/jpn.120152

Burgess, P. W., Alderman, N., Evans, J., Emslie, H., and Wilson, B. A. (1998). The ecological validity of tests of executive function. J. Int. Neuropsychol. Soc. 4, 547-558. doi: 10.1017/S1355617798466037
Burke, D. (2001). Empathy in sexually offending and nonoffending in adolescent males. J. Interpers. Violence 16, 222-233. doi: 10.1177/088626001016003003

Bush, C. A., Mullis, R. L., and Mullis, A. K. (2000). Differences between offender and nonoffender youth. J. Youth Adolesc. 29, 467-478. doi: 10.1023/A:1005162 526769

Cardona, J. F., Sinay, V., Amoruso, L., Hesse, E., Manes, F., and Ibanez, A. (2014). The impact of neuromyelitis optica on the recognition of emotional facial expressions: a preliminary report. Soc. Neurosci. 9, 633-638. doi: 10.1080/17470919.2014.935474

Carswell, K., Maughan, B., Davis, H., Davenport, F., and Goddard, N. (2004). The psychosocial needs of young offenders and adolescents from an inner city area. J. Adolesc. 27, 415-428. doi: 10.1016/j.adolescence.2004.04.003

Catell, R. (1971). Abilities: Their Structure, Growth and Action. Boston: HoughtonMifflin.

Cima, M., Tonnaer, F., and Hauser, M. D. (2010). Psychopaths know right from wrong but don't care. Soc. Cogn. Affect. Neurosci. 5, 59-67. doi: $10.1093 /$ scan/nsp051

Couto, B., Sedeno, L., Sposato, L. A., Sigman, M., Riccio, P. M., Salles, A., et al. (2013). Insular networks for emotional processing and social cognition: comparison of two case reports with either cortical or subcortical involvement. Cortex 49, 14201434. doi: 10.1016/j.cortex.2012.08.006

Davis, M. (1983). Measuring individual differences in empathy: evidence for a multidimensional approach. J. Pers. Soc. Psychol. 44, 113-126. doi: 10.1037/00223514.44.1.113

Decety, J., Michalska, K. J., Akitsuki, Y., and Lahey, B. B. (2009). Atypical empathic responses in adolescents with aggressive conduct disorder: a functional MRI investigation. Biol. Psychol. 80, 203-211. doi: 10.1016/j.biopsycho.2008. 09.004

Decety, J., Michalska, K. J., and Kinzler, K. D. (2012a). The contribution of emotion and cognition to moral sensitivity: a neurodevelopmental study. Cereb. Cortex 22, 209-220. doi: 10.1093/cercor/bhr111

Decety, J., Norman, G. J., Berntson, G. G., and Cacioppo, J. T. (2012b). A neurobehavioral evolutionary perspective on the mechanisms underlying empathy. Prog. Neurobiol. 98, 38-48. doi: 10.1016/j.pneurobio.2012.05.001

Decety, J., Skelly, L. R., and Kiehl, K. A. (2013). Brain response to empathy-eliciting scenarios involving pain in incarcerated individuals with psychopathy. JAMA Psychiatry 70, 638-645. doi: 10.1001/jamapsychiatry.2013.27

de Wied, M., van Boxtel, A., Matthys, W., and Meeus, W. (2012). Verbal, facial and autonomic responses to empathy-eliciting film clips by disruptive male adolescents with high versus low callous-unemotional traits. J. Abnorm. Child Psychol. 40, 211-223. doi: 10.1007/s10802-011-9557-8

Domes, G., Hollerbach, P., Vohs, K., Mokros, A., and Habermeyer, E. (2013). Emotional empathy and psychopathy in offenders: an experimental study. J. Personal. Disord. 27, 67-84. doi: 10.1521/pedi.2013.27.1.67

Ekman, P., and Friesen, E. (1976). Pictures of Facial Affects. Palo Alto, CA: Consulting Psychologists Press.

Ellis, P. L. (1982). Empathy: a factor in antisocial behavior. J. Abnorm. Child Psychol. 10, 123-134. doi: 10.1007/BF00915957

Escobar, M. J., Huepe, D., Decety, J., Sedeno, L., Messow, M. K., Baez, S., et al. (2014). Brain signatures of moral sensitivity in adolescents with early social deprivation. Sci. Rep. 4, 5354. doi: 10.1038/srep05354

Fairchild, G., Van Goozen, S. H., Calder, A. J., Stollery, S. J., and Goodyer, I. M. (2009). Deficits in facial expression recognition in male adolescents with early-onset or adolescence-onset conduct disorder. J. Child Psychol. Psychiatry 50, 627-636. doi: 10.1111/j.1469-7610.2008.02020.x

Fiorentino, N., Gleichgerrcht, E., Roca, M., Cetkovich, M., Manes, F., and Torralva, T. (2013). The INECO Frontal Screening tool differentiates behavioral variant - frontotemporal dementia (bv-FTD) from major depression. Dement. Neuropsychol. 7, 33-39.

Frisell, T., Pawitan, Y., and Langstrom, N. (2012). Is the association between general cognitive ability and violent crime caused by family-level confounders? PLOS ONE 7:e41783. doi: 10.1371/journal.pone.0041783

Glass, S. J., and Newman, J. P. (2006). Recognition of facial affect in psychopathic offenders. J. Abnorm. Psychol. 115, 815-820. doi: 10.1037/0021-843X.115. 4.815

Glass, S. J., and Newman, J. P. (2009). Emotion processing in the criminal psychopath: the role of attention in emotion-facilitated memory. J. Abnorm. Psychol. 118, 229-234. doi: 10.1037/a0014866 
Gleichgerrcht, E., Chade, A., Torralva, T., Roca, M., and Manes, F. (2011). Comparing the neuropsychiatric profile of patients with Alzheimer Disease who present spared versus impaired executive functioning. Curr. Gerontol. Geriatr. Res. 2011, 514059. doi: 10.1155/2011/514059

Greenwald, A. G., McGhee, D. E., and Schwartz, J. L. (1998). Measuring individual differences in implicit cognition: the implicit association test. J. Pers. Soc. Psychol 74, 1464-1480. doi: 10.1037/0022-3514.74.6.1464

Hair, J., Black, G., Barry, B., and Anderson, R. (2010). Multivariate Data Analysis. Upper Saddle River, NJ: Pearson Prentice Hall.

Huepe, D., Roca, M., Salas, N., Canales-Johnson, A., Rivera-Rei, A. A., Zamorano, L., et al. (2011). Fluid intelligence and psychosocial outcome: from logical problem solving to social adaptation. PLOS ONE 6:e24858. doi: 10.1371/journal.pone. 0024858

Ibáñez, A. (2010). Early neural markers of implicit attitudes: N170 modulated by intergroup and evaluative contexts in IAT. Front. Hum. Neurosci. 4:188. doi 10.3389/fnhum.2010.00188

Ibanez, A., Aguado, J., Baez, S., Huepe, D., Lopez, V., Ortega, R., et al. (2014a). From neural signatures of emotional modulation to social cognition: individual differences in healthy volunteers and psychiatric participants. Soc. Cogn. Affect. Neurosci. 9, 939-950. doi: 10.1093/scan/nst067

Ibanez, A., Kotz, S. A., Barrett, L., Moll, J., and Ruz, M. (2014b). Situated affective and social neuroscience. Front. Hum. Neurosci. 8:547. doi: 10.3389/fnhum.2014.00547

Ibanez, A., Huepe, D., Gempp, R., Gutiérrez, V., Rivera-Rei, A., and Toledo, M. (2013). Empathy, sex and fluid intelligence as predictors of theory of mind. Pers. Individ. Dif. 54, 616-621. doi: 10.1016/j.paid.2012.11.022

Ibanez, A., Hurtado, E., Riveros, R., Urquina, H., Cardona, J. F., Petroni, A., et al. (2011). Facial and semantic emotional interference: a pilot study on the behavioral and cortical responses to the dual valence association task. Behav. Brain Funct. 7 8. doi: 10.1186/1744-9081-7-8

Ibáñez, A., and Manes, F. (2012). Contextual social cognition and the behavioral variant of frontotemporal dementia. Neurology 78, 1354-1362. doi: 10.1212/WNL.0b013e3182518375

Ickes, W. (2009). "Empathic accuracy: its links to clinical, cognitive, developmental, social, and physiological psychology," In The Social Neuroscience of Empathy, ed J. Decety and W. Ickes (Massachussets: Massachusetts Institute of Technology) 57-70.

Isen, J. (2010). A meta-analytic assessment of Wechsler's P > V sign in antisocial populations. Clin. Psychol. Rev. 30, 423-435. doi: 10.1016/j.cpr.2010.02.003

Jolliffe, D., and Farrington, D. (2004). Empathy and offending: a systematic review and meta-analysis. Aggress. Violent Behav. 9, 441-476. doi: 10.1016/j.avb.2003.03.001

Jones, A. P., Forster, A. S., and Skuse, D. (2007). What do you think you're looking at? Investigating social cognition in young offenders. Crim. Behav. Ment. Health 17, 101-106. doi: 10.1002/cbm.641

Jones, A. P., Happe, F. G., Gilbert, F., Burnett, S., and Viding, E. (2010). Feeling, caring, knowing: different types of empathy deficit in boys with psychopathic tendencies and autism spectrum disorder. J. Child Psychol. Psychiatry 51, 1188 1197. doi: 10.1111/j.1469-7610.2010.02280.x

Kaplan, P. J., and Arbuthnot, J. (1985). Affective empathy and cognitive role-taking in delinquent and nondelinquent youth. Adolescence 20, 323-333.

Kennedy, D. P., and Adolphs, R. (2012). The social brain in psychiatric and neurological disorders. Trends Cogn. Sci. (Regul. Ed.) 16, 559-572. doi: 10.1016/j.tics.2012.09.006

Kennedy, T. D., Burnett, K. F., and Edmonds, W. A. (2011). Intellectual, behavioral, and personality correlates of violent vs. non-violent juvenile offenders. Aggress. Behav. 37, 315-325. doi: 10.1002/ab.20393

Kipps, C. M., Nestor, P. J., Acosta-Cabronero, J., Arnold, R., and Hodges, J. R. (2009) Understanding social dysfunction in the behavioural variant of frontotemporal dementia: the role of emotion and sarcasm processing. Brain 132, 592-603. doi: 10.1093/brain/awn314

Koolhof, R., Loeber, R., Wei, E. H., Pardini, D., and D’Escury, A. C. (2007). Inhibition deficits of serious delinquent boys of low intelligence. Crim. Behav. Ment. Health 17, 274-292. doi: 10.1002/cbm.661

Lee, M., and Prentice, N. M. (1988). Interrelations of empathy, cognition, and moral reasoning with dimensions of juvenile delinquency. J. Abnorm. Child Psychol. 16, 127-139. doi: 10.1007/BF00913589

Lindsey, R., Carlozzi, A., and Eells, G. (2001). Differences in dispositional empathy of juvenile sex offenders, non-sex-offending delinquent juveniles, and nondelinquent juveniles. J. Interpers. Violence 16, 510-521. doi: 10.1177/088626001016006002

Lockwood, P. L., Bird, G., Bridge, M., and Viding, E. (2013a). Dissecting empathy: high levels of psychopathic and autistic traits are characterized by difficulties in different social information processing domains. Front. Hum. Neurosci. 7:760. doi: 10.3389/fnhum.2013.00760

Lockwood, P. L., Sebastian, C. L., McCrory, E. J., Hyde, Z. H., Gu, X., De Brito, S., et al. (2013b). Association of callous traits with reduced neural response to others' pain in children with conduct problems. Curr. Biol. 23, 901-905. doi: 10.1016/j.cub.2013.04.018

Lovett, B. J., and Sheffield, R. A. (2007). Affective empathy deficits in aggressive children and adolescents: a critical review. Clin. Psychol. Rev. 27, 1-13. doi: 10.1016/j.cpr.2006.03.003

Mancini, G., Agnoli, S., Baldaro, B., Bitti, P. E., and Surcinelli, P. (2013). Facial expressions of emotions: recognition accuracy and affective reactions during late childhood. J. Psychol. 147, 599-617. doi: 10.1080/00223980.2012. 727891

Marsh, A. A., and Blair, R. J. (2008). Deficits in facial affect recognition among antisocial populations: a meta-analysis. Neurosci. Biobehav. Rev. 32, 454-465. doi: 10.1016/j.neubiorev.2007.08.003

Marsh, A. A., Finger, E. C., Fowler, K. A., Adalio, C. J., Jurkowitz, I. T., Schechter, J. C., et al. (2013). Empathic responsiveness in amygdala and anterior cingulate cortex in youths with psychopathic traits. J. Child Psychol. Psychiatry 54, 900-910. doi: $10.1111 /$ jcpp. 12063

McCown, W., Johnson, J., and Austin, S. (1986). Inability of delinquents to recognize facial affect. J. Soc. Behav. Pers. 1, 489-494.

McDonald, S., Bornhofen, C., Shum, D., Long, E., Saunders, C., and Neulinger, K. (2006). Reliability and validity of the awareness of social inference test (TASIT): a clinical test of social perception. Disabil. Rehabil. 28, 1529-1542. doi: 10.1080/09638280600646185

Melloni, M., Lopez, V., and Ibanez, A. (2014). Empathy and contextual social cognition. Cogn. Affect. Behav. Neurosci. 14, 407-425. doi: 10.3758/s13415-0130205-3

Mottus, R., Guljajev, J., Allik, J., Laidra, K., and Pullmann, H. (2012). Longitudinal associations of cognitive ability, personality traits and school grades with antisocial behaviour. Eur. J. Pers. 26, 56-62. doi: 10.1002/per.820

Newman, J. P., Curtin, J. J., Bertsch, J. D., and Baskin-Sommers, A. R. (2010). Attention moderates the fearlessness of psychopathic offenders. Biol. Psychiatry 67, 66-70. doi: 10.1016/j.biopsych.2009.07.035

Pardini, D. A., and Fite, P. J. (2010). Symptoms of conduct disorder, oppositional defiant disorder, attention-deficit/hyperactivity disorder, and callousunemotional traits as unique predictors of psychosocial maladjustment in boys: advancing an evidence base for DSM-V. J. Am. Acad. Child Adolesc. Psychiatry 49, 1134-1144. doi: 10.1016/j.jaac.2010.07.010

Parra, M. A., Sánchez, L. J., Rivera, C. M., and Arango, J. C. (2005). Evidencias preliminares de disfunción ejecutiva en homicidas no psicópatas. Anuario de Psicología Jurídica, 15, 97-107.

Pasalich, D. S., Dadds, M. R., and Hawes, D. J. (2014). Cognitive and affective empathy in children with conduct problems: additive and interactive effects of callous-unemotional traits and autism spectrum disorders symptoms. Psychiatry Res. 219, 625-630. doi: 10.1016/j.psychres.2014.06.025

Pham, T. H., and Philippot, P. (2010). Decoding of facial expression of emotion in criminal psychopaths. J. Personal. Disord. 24, 445-459. doi: 10.1521/pedi.2010.24.4.445

Radke, S., Brazil, I. A., Scheper, I., Bulten, B. H., and de Bruijn, E. R. (2013). Unfair offers, unfair offenders? Fairness considerations in incarcerated individuals with and without psychopathy. Front. Hum. Neurosci. 7:406. doi: 10.3389/fnhum.2013.00406

Rankin, K. P., Salazar, A., Gorno-Tempini, M. L., Sollberger, M., Wilson, S. M., Pavlic, D., et al. (2009). Detecting sarcasm from paralinguistic cues: anatomic and cognitive correlates in neurodegenerative disease. Neuroimage 47, 2005-2015. doi: 10.1016/j.neuroimage.2009.05.077

Raven, J., Court, J., and Raven, J. (Eds ). (2008). Test De Matrices Progresivas. Manual. Escalas Coloreadas, General y Avanzada. Buenos Aires: Paidos.

Sadeh, N., Spielberg, J. M., Heller, W., Herrington, J. D., Engels, A. S., Warren, S. L., et al. (2013). Emotion disrupts neural activity during selective attention in psychopathy. Soc. Cogn. Affect. Neurosci. 8, 235-246. doi: 10.1093/scan/n sr092 
Sato, W., Uono, S., Matsuura, N., and Toichi, M. (2009). Misrecognition of facial expressions in delinquents. Child Adolesc. Psychiatry Ment. Health 3, 27. doi: 10.1186/1753-2000-3-27

Schmoll, D. (2012). [Decision making, empathy and morality in psychopaths: does empirical research offer new perspectives concerning legal responsibility?] Fortschr. Neurol. Psychiatr. 80, 193-200. doi: 10.1055/s-0031-1281698

Schwenck, C., Mergenthaler, J., Keller, K., Zech, J., Salehi, S., Taurines, R., et al (2012). Empathy in children with autism and conduct disorder: group-specific profiles and developmental aspects. J. Child Psychol. Psychiatry 53, 651-659. doi: 10.1111/j.1469-7610.2011.02499.x

Sebastian, C. L., McCrory, E. J., Cecil, C. A., Lockwood, P. L., De Brito, S. A., Fontaine, N. M., et al. (2012). Neural responses to affective and cognitive theory of mind in children with conduct problems and varying levels of callous-unemotional traits. Arch. Gen. Psychiatry 69, 814-822. doi: 10.1001/archgenpsychiatry.2011.2070

Thomas, L. A., De Bellis, M. D., Graham, R., and LaBar, K. S. (2007). Development of emotional facial recognition in late childhood and adolescence. Dev. Sci. 10 547-558. doi: 10.1111/j.1467-7687.2007.00614.x

Torralva, T., Gleichgerrcht, E., Lischinsky, A., Roca, M., and Manes, F. (2012). "Ecological" and highly demanding executive tasks detect real life deficitsin high functioning adult ADHD patients. J. Atten. Disord. 17, 11-19. doi: $10.1177 / 1087054710389988$

Torralva, T., Roca, M., Gleichgerrcht, E., Bekinschtein, T., and Manes, F. (2009a). A neuropsychological battery to detect specific executive and social cognitive impairments in early frontotemporal dementia. Brain 132, 1299-1309. doi: 10.1093/brain/awp041

Torralva, T., Roca, M., Gleichgerrcht, E., Lopez, P., and Manes, F. (2009b). INECO Frontal Screening (IFS): a brief, sensitive, and specific tool to assess executive functions in dementia. J. Int. Neuropsychol. Soc. 15, 777-786. doi: $10.1017 /$ S1355617709990415
Verona, E., Sprague, J., and Sadeh, N. (2012). Inhibitory control and negative emotional processing in psychopathy and antisocial personality disorder. J. Abnorm. Psychol. 121, 498-510. doi: 10.1037/a0025308

Viding, E., Sebastian, C. L., Dadds, M. R., Lockwood, P. L., Cecil, C. A., De Brito, S. A., et al. (2012). Amygdala response to preattentive masked fear in children with conduct problems: the role of callous-unemotional traits. Am. J. Psychiatry 169, 1109-1116. doi: 10.1176/appi.ajp.2012.12020191

Young, A. W., Rowland, D., Calder, A. J., Etcoff, N. L., Seth, A., and Perrett, D. I. (1997). Facial expression megamix: tests of dimensional and category accounts of emotion recognition. Cognition 63, 271-313. doi: 10.1016/S0010-0277(97) 00003-6

Conflict of Interest Statement: The authors declare that the research was conducted in the absence of any commercial or financial relationships that could be construed as a potential conflict of interest.

Received: 22 July 2014; accepted: 03 October 2014; published online: 21 October 2014 Citation: Gonzalez-Gadea ML, Herrera E, Parra M, Gomez Mendez P, Baez S, Mane $F$ and Ibanez A (2014) Emotion recognition and cognitive empathy deficits in adolescent offenders revealed by context-sensitive tasks. Front. Hum. Neurosci. 8:850. doi 10.3389/fnhum.2014.00850

This article was submitted to the journal Frontiers in Human Neuroscience.

Copyright (C) 2014 Gonzalez-Gadea, Herrera, Parra, Gomez Mendez, Baez, Manes and Ibanez. This is an open-access article distributed under the terms of the Creative Commons Attribution License (CC BY). The use, distribution or reproduction in other forums is permitted, provided the original author(s) or licensor are credited and that the original publication in this journal is cited, in accordance with accepted academic practice. No use, distribution or reproduction is permitted which does not comply with these terms. 